\title{
Equações de chuvas intensas para o Estado do Pará
}

\author{
Rodrigo O. R. de M. Souza1, Paulo H. M. Scaramussa1, Marcos A. C. M. do Amaral ${ }^{2}$, \\ J. A. Pereira Neto ${ }^{1}$, Alexandre V. Pantoja ${ }^{1}$ \& Luis W. R. Sadeck ${ }^{3}$
}

\begin{abstract}
RESUMO
As equações de chuvas intensas têm sido usadas como ferramenta importante para o dimensionamento de obras hidráulicas. Devido à grande carência de informações relativas às equações de chuvas intensas, o presente trabalho teve como objetivo a obtenção das relações de intensidade, duração e frequência de precipitação pluvial para o Estado do Pará, utilizando-se a metodologia da desagregação da chuva de 24 h. Foram utilizadas séries históricas de dados pluviométricos de 74 cidades do Estado do Pará, obtidas no Sistema de Informações Hidrológicas da Agência Nacional de Águas-ANA. As equações de intensidadeduração-frequência foram devidamente ajustadas e apresentaram bom ajuste, com coeficientes de determinação acima de 0,99. A maioria das estações $(51,4 \%)$ apresentou intensidade de precipitação entre 90 e $110 \mathrm{~mm} \mathrm{~h}^{-1}$, para uma duração de chuva de $30 \mathrm{~min}$ e um tempo de retorno de 15 anos. Podese perceber uma concentração das maiores precipitações na região próxima ao litoral do nordeste paraense e no sudeste da Ilha do Marajó.
\end{abstract}

Palavras-chave: precipitação, desagregação da chuva de 24 h, intensidade-duração-frequência, hidrologia

\section{Intense rainfall equations for the State of Pará, Brazil}

\section{ABSTRACT}

The intense rainfall equations have been used as an important tool for design of hydraulic structures. Considering the lack of intense rainfall equations, this study aimed to determine the relations of intensity, duration and frequency of intense rainfall in the Pará State (Brazil), using the one-day rain disaggregation method. In this research rainfall data of 74 cities in the State of Pará were used, obtained from the Hydrological Information System of the National Water Agency-ANA. The equations of intensity-durationfrequency were adjusted and presented good adjustment with coefficients of determination above 0.99. Most stations $\left(51.4 \%\right.$ ) showed intensity of precipitation between 90 and $110 \mathrm{~mm} \mathrm{~h}^{-1}$ for duration of 30 min and rainfall return period of 15 years. The highest rainfall intensities were in the region near the northeast coast of Pará State and southeast of the Marajo Island.

Key words: rain, one-day rain disaggregation, intensity-duration-frequency, hydrology 


\section{INTRODUÇÃO}

Devido ao histórico de ocupação agrícola do estado do Pará muitas bacias hidrográficas estão em processo de degradação. Entretanto, poucas são as informações técnicas sobre as características climáticas e físico-hídricas dos solos dessas regiões, que possam contribuir para a redução da degradação.

No planejamento do uso da água e do solo é indispensável dispor de informações relacionadas às variáveis climáticas, tal como as chuvas intensas. A caracterização da variabilidade temporal das chuvas intensas ao longo de sua duração é imprescindível para quantificar adequadamente os efeitos ocasionados no escoamento superficial em áreas urbanas e rurais (Silva \& Clarke, 2004).

As equações de chuvas intensas têm sido usadas como ferramenta importante para a elaboração de projetos de obras hidráulicas, como dimensionamento de vertedores, retificação de cursos d água, galerias de águas pluviais, bueiros, sistemas de drenagem agrícola, urbana e rodoviária (Beijo et al., 2003; Mello et al., 2008; Oliveira et al., 2008a).

As chuvas intensas são caracterizadas por suas intensidade (I), duração (D) e frequência $(F)$ de ocorrência, podendo ser representadas por equações denominadas IDF. Para a obtenção dessas equações são necessários dados pluviográficos e, segundo Genovez \& Zuffo (2000), apresentam validade regional. Santos et al. (2009) também confirmam o caráter local das equações de IDF e acrescentam a importância da utilização de séries longas de dados observados para a estimativa mais eficiente das equações.

A determinação das equações IDF por meio de dados pluviográficos apresenta grande dificuldade em razão da baixa densidade de pontos de coleta e do pequeno período de observações normalmente disponíveis.

Por esta razão, em algumas regiões poucos trabalhos têm sido realizados com tal finalidade ocasionando grande entrave na realização de projetos de obras hidráulicas mais confiáveis e econômicos.

O trabalho clássico de estudos da relação IntensidadeDuração-Frequência (IDF) da precipitação pluvial no Brasil foi publicado por Pfafstetter (1957) para 98 localidades, com base em dados dos postos do serviço de meteorologia do Ministério da Agricultura. Outros trabalhos de determinação de equações de IDF foram de: Pinto et al. (1999) e Mello et al. (2003) para o Estado de Minas Gerais; Silva et al. (2003), para o estado do Tocantins; Oliveira et al. (2005, 2008b), para o estado de Goiás e Distrito Federal; Silva et al. (1999) para o estado do Rio de Janeiro e Espírito Santo, Back (2009) para Urussanga, SC, e Longo et al. (2006) para Cascavel, PR.

O estado do Pará caracteriza-se pela carência de dados pluviográficos, o que justifica a falta de dados bibliográficos sobre equações de chuvas intensas no Estado. Uma alternativa para a estimativa dessas equações seria a utilização de dados pluviométricos, visto que o Estado possui grande número de pluviômetros.

Algumas metodologias foram desenvolvidas no Brasil com vista à obtenção de chuvas de menor duração a partir de registros pluviométricos diários devido à existência no território nacional de vasta rede pluviométrica. Tais metodologias empregam coeficientes para transformar chuva de $24 \mathrm{~h}$, em chuvas de menor duração, dentre as quais estão a das isozonas e a da desagregação da chuva de $24 \mathrm{~h}$.

Damé et al. (2008) realizaram uma comparação entre diferentes metodologias para estimativa de curvas IDF. Dentre as metodologias estudadas a de desagregação de chuvas de $24 \mathrm{~h}$ com os coeficientes propostos pelo DAEE-CETESB (1980) foi a que melhor representou as intensidades máximas de precipitação.

Robaina (1996) avaliou a técnica da desagregação de chuvas de 24 h para 32 localidades do estado do Rio Grande do Sul e recomenda o uso da metodologia em localidades onde não há disponibilidade de registros pluviográficos.

Devido à grande carência de informações relativas às equações de chuvas intensas no Estado do Pará, o presente trabalho teve como objetivo a obtenção das relações de intensidade, duração e frequência de chuvas intensas para 74 estações pluviométricas do Estado, através da metodologia da desagregação da chuva de 24 h.

\section{Material e MÉTODOS}

Neste trabalho foram utilizadas séries históricas de dados pluviométricos do estado do Pará, com no mínimo 10 anos de registro. Os dados foram obtidos no Sistema de Informação Hidrológica da Agência Nacional de Águas (ANA, 2011), perfazendo 74 estações pluviométricas (Figura 1). Para cada estação foram elaboradas as séries históricas dos valores máximos anuais de precipitações diárias.

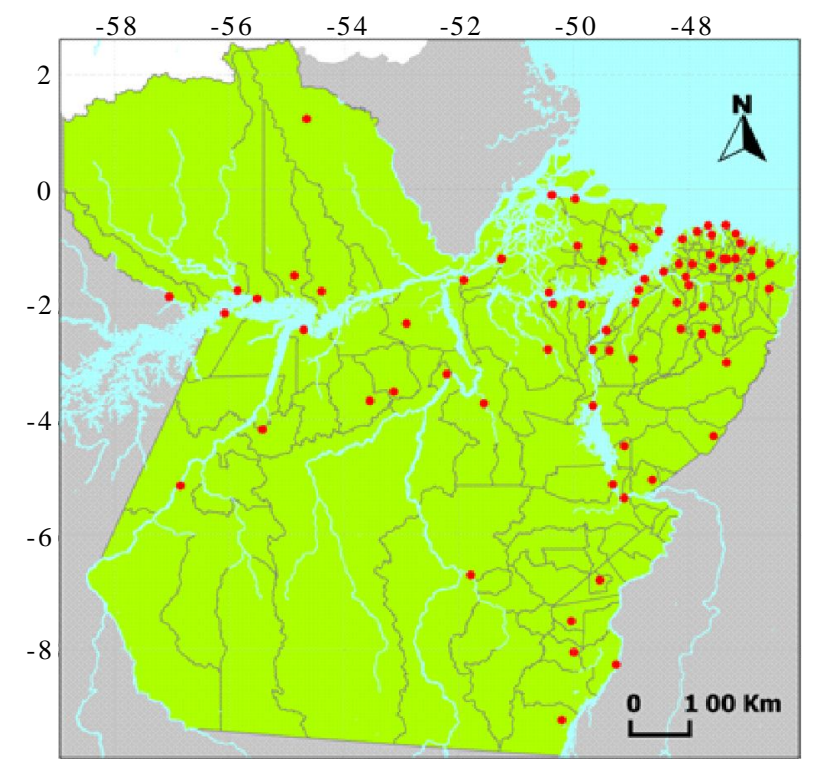

Figura 1. Localização das estações pluviométricas no Estado do Pará

O banco de dados da ANA utiliza informações de diferentes fontes: Instituto Nacional de Meteorologia (INMET), Serviço Geológico do Brasil (CPRM) e Secretaria de Meio Ambiente do Estado do Pará (SEMA). A partir deste banco de dados 
foram obtidos os valores máximos anuais de precipitação diária.

Os dados de precipitação máxima foram ajustados ao modelo de distribuição de Gumbel. Após a verificação da aderência dos dados à distribuição de Gumbel para cada série de duração de chuva realizaram-se as estimativas das chuvas máximas para períodos de retorno de 5, 10, 20, 50, 100, 1.000 e 10.000 anos. A análise de aderência da distribuição de Gumbel foi feita pelo teste de Kolmogorov-Smirnov.

As distribuições de valores extremos de grandezas hidrológicas se ajustam satisfatoriamente à distribuição de Gumbel, empregada neste trabalho. Segundo Silva et al. (2002) o modelo de Gumbel foi o que apresentou melhor ajuste aos dados de intensidades máximas médias de precipitação pluvial pelo teste de Kolmogorov-Smirnov, a 20\% de probabilidade para todas as durações estudadas.

O modelo de distribuição de probabilidades de Gumbel foi utilizado para o cálculo anual das precipitações máximas diárias por meio da seguinte sequência de equações, conforme Pinto (1995) (Eqs. 1, 2 e 3):

$$
\begin{gathered}
\mathrm{Y}_{\mathrm{TR}}=-\ln \left[-\ln \left(1-\frac{1}{\mathrm{TR}}\right)\right] \\
\mathrm{K}_{\mathrm{TR}}=-0,45+0,78 \times \mathrm{Y}_{\mathrm{TR}} \\
\mathrm{X}_{\mathrm{TR}}=\overline{\mathrm{X}}+\mathrm{K}_{\mathrm{TR}} \times \mathrm{S}
\end{gathered}
$$

em que:

$\mathrm{Y}_{\mathrm{TR}}$ - variável reduzida da distribuição de Gumbel

TR - período de retorno, anos

$\mathrm{X}_{\mathrm{TR}}$ - precipitação máxima diária para determinado $\mathrm{TR}, \mathrm{mm}$

$\mathrm{K}_{\mathrm{TR}}$ - fator de frequência, adimensional

$\underline{X}$ eS - média da precipitação máxima diária, mm, e o desviopadrão dos dados de precipitação máxima diária, mm

Para o ajuste das equações de chuvas intensas foram utilizadas chuvas de diferentes durações. As precipitações máximas diárias obtidas para diferentes tempos de retorno foram desagregadas em chuvas com duração menor.

A desagregação da chuva de um dia em chuvas de menor duração foi obtida pela metodologia proposta pelo DAEECETESB (1980). As durações de 5, 10, 15, 20, 25, 30, 60, 360, 480, 600,720 e $1440 \mathrm{~min}$ foram utilizadas pelo emprego dos coeficientes multiplicativos, apresentados na Tabela 1 permitindo, desta forma, a geração de pontos suficientes para definir as

Tabela 1. Coeficiente de desagregação da chuva de $24 \mathrm{~h}$ de duração

\begin{tabular}{cccc}
\hline Duração & Coeficientes & Duração & Coeficientes \\
$24 \mathrm{~h} 24 \mathrm{~h}^{-1}$ & 1,14 & $30 \mathrm{~min} \mathrm{~h}^{-1}$ & 0,74 \\
$12 \mathrm{~h} 24 \mathrm{~h}^{-1}$ & 0,85 & $25{\operatorname{min~} 0,5 \mathrm{~h}^{-1}}^{-1}$ & 0,91 \\
$10 \mathrm{~h} 24 \mathrm{~h}^{-1}$ & 0,82 & $20 \operatorname{min~} 0,5 \mathrm{~h}^{-1}$ & 0,81 \\
$8 \mathrm{~h} 24 \mathrm{~h}^{-1}$ & 0,78 & $15 \mathrm{~min} 0,5 \mathrm{~h}^{-1}$ & 0,70 \\
$6 \mathrm{~h} 24 \mathrm{~h}^{-1}$ & 0,72 & $10 \operatorname{min~} 0,5 \mathrm{~h}^{-1}$ & 0,54 \\
$1 \mathrm{~h} 24 \mathrm{~h}^{-1}$ & 0,42 & $5 \operatorname{min~} 0,5 \mathrm{~h}^{-1}$ & 0,34 \\
\hline Fonte: DAEE/CETESB (1980) & & &
\end{tabular}

curvas de intensidade-duração referentes a diferentes períodos de retorno.

Com os valores obtidos de chuvas máximas para diferentes durações e tempos de retorno, estimaram-se os parâmetros da equação que expressa a relação IDF (Eq. 4), para cada estação observada.

$$
I=\frac{K \times T R^{a}}{(t+b)^{c}}
$$

em que:
I - intensidade de precipitação, $\mathrm{mm} \mathrm{h}^{-1}$
TR - período de retorno, anos
$\mathrm{t}$ - tempo de duração da chuva, min
$\mathrm{K}, \mathrm{a}, \mathrm{b}, \mathrm{c}$ - constantes

Os ajustes das equações de intensidade de precipitação foram realizados no software Table Curve 3D.

Para a avalição da distribuição espacial da intensidade de precipitação a mesma foi calculada considerando-se um tempo de retorno de 15 anos e duração de $30 \mathrm{~min}$ com as equações ajustadas de cada estação. Os dados foram espacializados no mapa do Pará com o software TerraView. A estimativa foi realizada para apenas uma duração de chuva e um tempo de retorno, pois não haveria mudanças espaciais representativas dos mapas para as diferentes durações.

Ressalta-se que a escolha do tempo de retorno para o dimensionamento de uma obra hidráulica está relacionada com a vida útil da obra, o tipo de estrutura, a facilidade de reparos e o perigo oferecido à vida humana. A escolha da duração da chuva em projetos de drenagem e barragens de terra está relacionada com o tempo de concentração da bacia de contribuição.

\section{RESULTADOS E DISCUSSÃO}

As 74 séries históricas de precipitação diária máxima anual utilizadas neste trabalho apresentaram duração média de 24 anos. A média das maiores chuvas de cada estação foi de 169,3 mm.

A estação do município de Soure possui a série histórica com maior média (150,1 mm) e a estação do município de Chaves possui a menor média $(67,1 \mathrm{~mm})$. A série histórica de 43 anos da estação de Belém apresentou o valor máximo de 136,9 mm (março de 1985) com média de $89,3 \mathrm{~mm}$ e desvio padrão de $20 \mathrm{~mm}$.

Para todos os municípios trabalhados o modelo de distribuição de Gumbel mostrou-se adequado para representar as estimativas dos valores de precipitação máxima.

Na Tabela 2 pode-se observar os parâmetros ajustados das equações de IDF, relativos às 74 estações pluviométricas no Estado do Pará identificadas pelos municípios onde estão instaladas. Para todas elas se verificam ajustes adequados, com valores $\mathrm{R}^{2}$ acima de 0,99 .

Santos et al. (2009) e Silva et al. (2003) realizaram trabalhos semelhantes para o Estado de Mato Grosso do Sul e Tocantins obtendo, respectivamente, coeficientes de determinação médios de 0,99 e 0,98 . 
Tabela 2. Coeficientes " $K$ ", "a", "b" e " $c$ " das equações de chuvas intensas ajustadas para várias localidades do Estado do Pará e respectivos coeficientes de determinação $\left(R^{2}\right)$ e duração das séries históricas

\begin{tabular}{|c|c|c|c|c|c|c|}
\hline Município & $\bar{K}$ & $a$ & b & C & $\mathrm{R}^{2}$ & Séries Históricas (anos) \\
\hline Abaetetuba & 1086,3999 & 0,1193 & 9,7855 & 0,7242 & 0,9957 & 29 \\
\hline Acará & 1153,9489 & 0,0973 & 9,7945 & 0,7244 & 0,9978 & 27 \\
\hline Afuá & 688,6278 & 0,0505 & 9,8028 & 0,7247 & 0,9995 & 10 \\
\hline Alenquer & 952,4028 & 0,1078 & 9,7929 & 0,7244 & 0,9969 & 34 \\
\hline Almeirim & 1031,0537 & 0,1095 & 9,7988 & 0,7245 & 0,9968 & 30 \\
\hline Altamira & 1204,0580 & 0,1152 & 9,7894 & 0,7243 & 0,9961 & 28 \\
\hline Anajás & 1148,3197 & 0,1266 & 9,7913 & 0,7244 & 0,9947 & 11 \\
\hline Augusto Corrêa & 1109,8761 & 0,1056 & 9,7878 & 0,7242 & 0,9971 & 24 \\
\hline Aurora do Pará & 1130,3284 & 0,1175 & 9,7859 & 0,7242 & 0,9959 & 22 \\
\hline Aveiro & 1003,1550 & 0,0930 & 9,7925 & 0,7244 & 0,9981 & 29 \\
\hline Bagre & 1283,2098 & 0,1280 & 9,7886 & 0,7242 & 0,9945 & 25 \\
\hline Baião & 1268,3666 & 0,1114 & 9,7940 & 0,7244 & 0,9966 & 18 \\
\hline Barcarena & 1007,3605 & 0,1070 & 9,7931 & 0,7243 & 0,9970 & 23 \\
\hline Belém & 960,5846 & 0,0954 & 9,7993 & 0,7245 & 0,9979 & 43 \\
\hline Bom Jesus do Tocantins & 1036,5976 & 0,1017 & 9,7840 & 0,7242 & 0,9974 & 11 \\
\hline Bragança & 1206,4232 & 0,1087 & 9,7844 & 0,7242 & 0,9968 & 19 \\
\hline Breves & 843,3295 & 0,0985 & 9,7958 & 0,7244 & 0,9977 & 21 \\
\hline Bujaru & 815,3742 & 0,0920 & 9,7957 & 0,7243 & 0,9981 & 16 \\
\hline Cametá & 1216,6448 & 0,0951 & 9,7918 & 0,7244 & 0,9979 & 21 \\
\hline Capanema & 1136,7892 & 0,1163 & 9,7866 & 0,7242 & 0,9960 & 43 \\
\hline Castanhal & 1216,7335 & 0,1266 & 9,7886 & 0,7243 & 0,9947 & 31 \\
\hline Chaves & 763,3059 & 0,1115 & 9,7930 & 0,7244 & 0,9965 & 11 \\
\hline Conceição do Araguaia & 865,9467 & 0,0959 & 9,7969 & 0,7246 & 0,9979 & 29 \\
\hline Curuçá & 1399,1103 & 0,0997 & 9,7918 & 0,7243 & 0,9976 & 27 \\
\hline Dom Eliseu & 1128,4211 & 0,1059 & 9,8007 & 0,7246 & 0,9971 & 27 \\
\hline Faro & 1038,5272 & 0,1038 & 9,7916 & 0,7244 & 0,9973 & 20 \\
\hline Gurupá & 896,3305 & 0,1096 & 9,7851 & 0,7243 & 0,9967 & 18 \\
\hline Igarapé-Açu & 1131,1570 & 0,1121 & 9,7860 & 0,7242 & 0,9965 & 29 \\
\hline Igarapé-Miri & 1008,3703 & 0,0948 & 9,7850 & 0,7242 & 0,9980 & 13 \\
\hline Ipixuna do Pará & 1106,1890 & 0,1000 & 9,7919 & 0,7244 & 0,9976 & 24 \\
\hline Irituia & 1040,6804 & 0,1144 & 9,7933 & 0,7243 & 0,9962 & 28 \\
\hline Itaituba & 1073,2685 & 0,1317 & 9,7851 & 0,7242 & 0,9939 & 30 \\
\hline Itupiranga & 1242,7328 & 0,1111 & 9,7853 & 0,7242 & 0,9966 & 15 \\
\hline Jacundá & 1192,2027 & 0,0809 & 9,7905 & 0,7243 & 0,9987 & 16 \\
\hline Juruti & 1292,2966 & 0,1073 & 9,8015 & 0,7245 & 0,9970 & 26 \\
\hline Magalhães Barata & 1248,4018 & 0,1134 & 9,7921 & 0,7244 & 0,9963 & 15 \\
\hline Marabá & 1242,9246 & 0,1057 & 9,7849 & 0,7242 & 0,9971 & 20 \\
\hline Marapanim & 1453,3908 & 0,1225 & 9,7927 & 0,7244 & 0,9953 & 17 \\
\hline Medicilândia & 1065,0031 & 0,0930 & 9,7887 & 0,7243 & 0,9981 & 13 \\
\hline Melgaço & 886,3028 & 0,0921 & 9,7834 & 0,7241 & 09981 & 18 \\
\hline Mocajuba & 1151,4508 & 0,0960 & 9,7964 & 0,7244 & 0,9979 & 15 \\
\hline Мoju & 1162,7321 & 0,1023 & 9,7929 & 0,7244 & 0,9974 & 28 \\
\hline Monte Alegre & 999,0226 & 0,1130 & 9,7838 & 0,7241 & 0,9964 & 36 \\
\hline Nova Timboteua & 859,0256 & 0,1017 & 9,7875 & 0,7243 & 0,9974 & 15 \\
\hline Óbidos & 990,8133 & 0,1103 & 9,7782 & 0,7241 & 0,9967 & 53 \\
\hline Oeiras do Pará & 1029,6561 & 0,0936 & 9,7975 & 0,7244 & 0,9980 & 21 \\
\hline Oriximiná & 1111,7396 & 0,1063 & 9,7954 & 0,7244 & 0,9970 & 39 \\
\hline Ourém & 887,0707 & 0,1055 & 9,7948 & 0,7245 & 0,9971 & 20 \\
\hline Paragominas & 1125,9005 & 0,1232 & 9,7922 & 0,7244 & 0,9952 & 24 \\
\hline Ponta de Pedras & 1211,6537 & 0,1042 & 9,7930 & 0,7244 & 0,9972 & 11 \\
\hline Portel & 1112,9052 & 0,0998 & 9,7830 & 0,7241 & 0,9976 & 33 \\
\hline Porto de Moz & 1137,9609 & 0,1287 & 9,7963 & 0,7244 & 0,9944 & 23 \\
\hline Prainha & 978,4673 & 0,1110 & 9,7883 & 0,7244 & 0,9966 & 29 \\
\hline Primavera & 1164,6366 & 0,0863 & 9,7956 & 0,7244 & 0,9985 & 28 \\
\hline Redenção & 1156,4648 & 0,1038 & 9,7910 & 0,7243 & 0,9973 & 26 \\
\hline Rio Maria & 1085,4709 & 0,0831 & 9,7952 & 0,7244 & 0,9986 & 13 \\
\hline Salinópolis & 1474,9764 & 0,0903 & 9,7870 & 0,7243 & 0,9983 & 32 \\
\hline Santa Cruz do Arari & 968,3271 & 0,1032 & 9,7880 & 0,7242 & 0,9973 & 11 \\
\hline Santa Isabel do Pará & 1088,7772 & 0,1227 & 9,7854 & 0,7243 & 0,9953 & 29 \\
\hline Santa Luzia do Pará & 956,0860 & 0,1176 & 9,7877 & 0,7241 & 0,9959 & 16 \\
\hline Santa Maria do Pará & 1292,0533 & 0,1480 & 9,7904 & 0,7243 & 0,9911 & 19 \\
\hline Santana do Araguaia & 864,5309 & 0,1047 & 9,7907 & 0,7244 & 0,9972 & 23 \\
\hline Santarém & 1310,4147 & 0,1064 & 9,7941 & 0,7244 & 0,9970 & 41 \\
\hline
\end{tabular}


Continuação da Tabela 2

\begin{tabular}{lcccccc}
\hline \multicolumn{1}{c}{ Município } & $\mathbf{K}$ & $\mathbf{a}$ & $\mathbf{b}$ & $\mathbf{C}$ & $\mathbf{R}^{2}$ & Séries Históricas (anos) \\
São Domingos do Capim & 1068,8693 & 0,1055 & 9,7942 & 0,7244 & 0,9971 & 34 \\
São Felix do Xingu & 1030,0221 & 0,1094 & 9,7970 & 0,7244 & 0,9968 & 24 \\
São João de Pirabas & 1428,9522 & 0,1109 & 9,7935 & 0,7244 & 0,9966 & 21 \\
Senador José Porfirio & 1243,2061 & 0,1198 & 9,7828 & 0,7241 & 0,9956 & 15 \\
Soure & 1648,2807 & 0,1024 & 9,7886 & 0,7243 & 0,9974 & 38 \\
Tailândia & 1089,9101 & 0,1157 & 9,7896 & 0,7243 & 0,9961 & 16 \\
Tomé-Açu & 1038,3348 & 0,0833 & 9,7931 & 0,7244 & 0,9986 & 25 \\
Tucuruí & 1264,6709 & 0,1012 & 9,7852 & 0,7242 & 0,9975 & 23 \\
Vigia & 1071,4102 & 0,0921 & 9,7815 & 0,7241 & 0,9981 & 28 \\
Viseu & 873,5618 & 0,0928 & 9,7850 & 0,7242 & 0,9981 & 33 \\
Xinguara & 1023,8456 & 0,1081 & 9,7965 & 0,7244 & 0,9969 & \\
\hline
\end{tabular}

Dentre as constantes ajustadas do modelo o " $\mathrm{K}$ " foi a que apresentou maior variação: de 688,63 (Afuá) a 1.648,28 (Soure). Esses resultados indicam variação das intensidades de precipitação esperadas para diferentes regiões do Estado. Para o parâmetro "a", a variação observada foi de 0,0505 a 0,1480 , para Afuá e Santa Maria do Pará, respectivamente. Os parâmetros "b" e "c" apresentaram valores próximos da média de 9,79 e 0,72 , respectivamente.

$\mathrm{Na}$ Tabela 3 pode-se observar as intensidades de precipitação estimadas com as equações dos municípios, para um tempo de retorno de 15 anos e duração de 30 min.

A estação do município Soure apresentou a maior intensidade de precipitação $\left(150,9 \mathrm{~mm} \mathrm{~h}^{-1}\right)$ e a de Afuá a menor $(54,7$ $\mathrm{mm} \mathrm{h}^{-1}$ ). Na Figura 2 pode-se observar a distribuição espacial das precipitações estimadas no Estado do Pará.

Dentre as estações com maiores intensidades a maioria está concentrada na região próxima ao litoral do nordeste paraense (Marapanim, Castanhal, Santa Maria do Pará, Salinópolis, Curuçá e São João de Pirabas). Na Ilha de Marajó a estação do

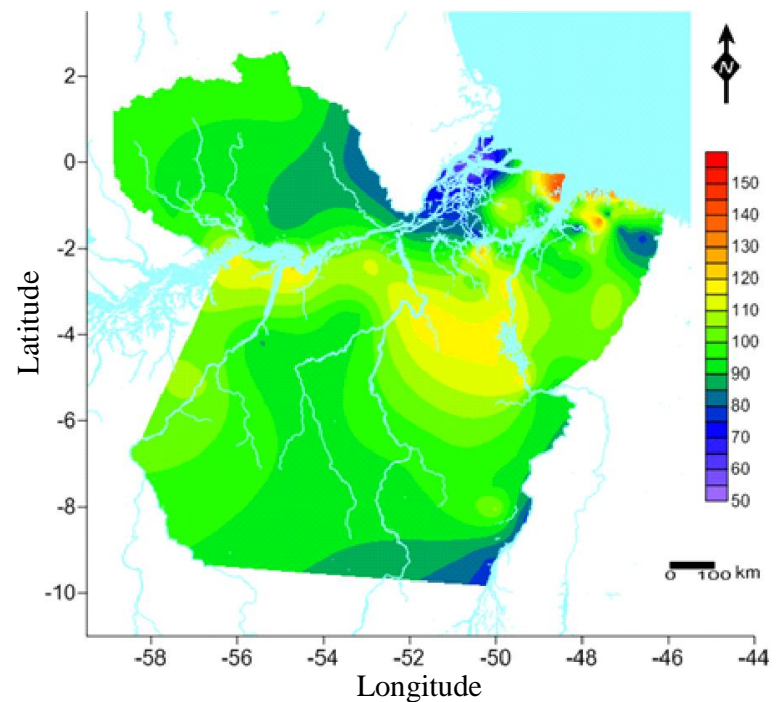

Figura 2. Distribuição espacial das intensidades de precipitação $\left(\mathrm{mm} \mathrm{h}^{-1}\right)$ com duração de 30 min e tempo de retorno de 15 anos para o Estado do Pará

Tabela 3. Intensidade de precipitação para uma duração de 30 min e um tempo de retorno de 15 anos para várias localidades do Estado do Pará

\begin{tabular}{|c|c|c|c|c|c|}
\hline Município & $\mathrm{I}\left(\mathrm{mm} \mathrm{h}^{-1}\right)$ & Município & $\mathrm{I}\left(\mathrm{mm} \mathrm{h}^{-1}\right)$ & Município & $\mathrm{I}\left(\mathrm{mm} \mathrm{h}^{-1}\right)$ \\
\hline Abaetetuba & 104,2 & Faro & 95,4 & Portel & 101,3 \\
\hline Acará & 104,2 & Gurupá & 83,7 & Porto de Moz & 111,8 \\
\hline Afuá & 54,7 & Igarapé-Açu & 106,4 & Prainha & 91,7 \\
\hline Alenquer & 88,5 & Igarapé-Miri & 90,5 & Primavera & 102,0 \\
\hline Almeirim & 96,2 & Ipixuna do Pará & 100,6 & Redenção & 106,3 \\
\hline Altamira & 114,1 & Irituia & 98,4 & Rio Maria & 94,3 \\
\hline Anajás & 112,2 & Itaituba & 106,4 & Salinópolis & 130,7 \\
\hline Augusto Corrêa & 102,5 & Itupiranga & 116,6 & Santa Cruz do Arari & 88,9 \\
\hline Aurora do Pará & 107,9 & Jacundá & 103,0 & Santa Isabel do Pará & 105,3 \\
\hline Aveiro & 89,5 & Juruti & 119,8 & Santa Luzia do Pará & 91,3 \\
\hline Bagre & 126,0 & Magalhães Barata & 117,7 & Santa Maria do Pará & 133,9 \\
\hline Baião & 118,9 & Marabá & 114,9 & Santana do Araguaia & 79,6 \\
\hline Barcarena & 93,4 & Marapanim & 140,5 & Santarém & 121,2 \\
\hline Belém & 86,2 & Medicilândia & 95,1 & São Domingos do Capim & 98,6 \\
\hline Bom Jesus do Tocantins & 94,8 & Melgaço & 79,0 & São Felix do Xingu & 96,1 \\
\hline Bragança & 112,4 & Mocajuba & 103,6 & São João de Pirabas & 133,8 \\
\hline Breves & 76,4 & Моји & 106,4 & Senados José Porfirio & 119,4 \\
\hline Bujaru & 72,6 & Monte Alegre & 94,2 & Soure & 150,9 \\
\hline Cametá & 109,2 & Nova Timboteua & 78,5 & Tailândia & 103,5 \\
\hline Capanema & 108,1 & Óbidos & 92,8 & Tomé-Açu & 90,2 \\
\hline Castanhal & 119,0 & Oeiras do Pará & 92,0 & Tucuruí & 115,5 \\
\hline Chaves & 71,6 & Oriximiná & 102,8 & Vigia & 95,5 \\
\hline Conceição do Araguaia & 77,8 & Ourém & 81,8 & Viseu & 78,0 \\
\hline Curuçá & 127,2 & Paragominas & 109,0 & Xinguara & 95,2 \\
\hline Dom Eliseu & 104,2 & Ponta de Pedras & 111,4 & & \\
\hline
\end{tabular}


município de Soure também apresentou elevado valor de intensidade de precipitação, o que se justifica pela proximidade do litoral e da costa do nordeste paraense.

A configuração espacial da zona de baixa pressão da linha do Equador proporciona os ventos alísios, responsáveis por transportar umidade das zonas tropicais para a zona equatorial, provocando chuvas nesta região.

As menores precipitações ficaram concentradas no noroeste da Ilha de Marajó (Melgaço, Breves, Chaves e Afuá) e na fronteira com o estado do Tocantins (Santana do Araguaia e Conceição do Araguaia).

Na Figura 3 observa-se a distribuição de frequência das estações conforme a intensidade de precipitação com duração de 30 min e tempo de retorno de 15 anos. A maioria das estações $(51,4 \%)$ apresentou intensidades entre 90 e $110 \mathrm{~mm} \mathrm{~h}^{-1}$.

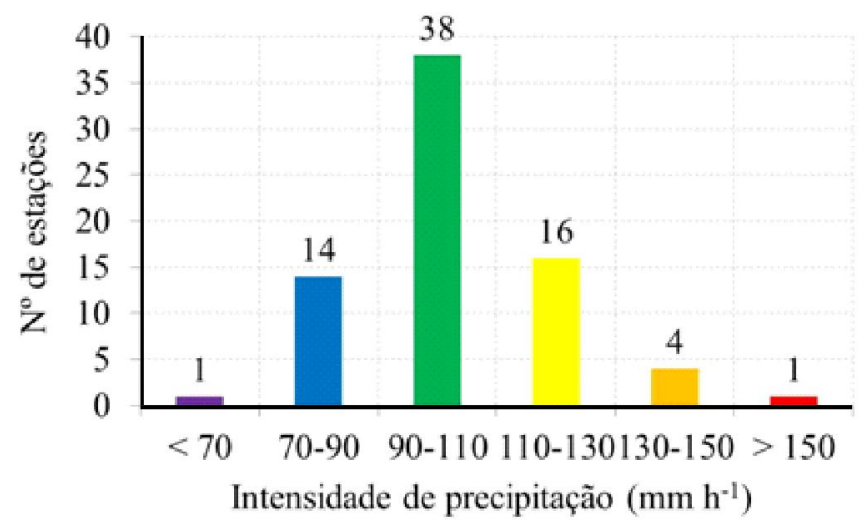

Figura 3. Distribuição de frequência das estações pluviométricas, conforme a intensidade de precipitação para o Estado do Pará

Em trabalhos realizados em outros estados considerando a mesma duração e tempo de retorno, os maiores valores obtidos de intensidade de precipitação foram: 119,5 $\mathrm{mm} \mathrm{h}^{-1} \mathrm{em}^{\text {Argoim, }}$ BA (Silva et al., 2002); 170,7 $\mathrm{mm} \mathrm{h}^{-1}$ em Cuiabá, MS (Santos et al., 2009); 181,9 $\mathrm{mm} \mathrm{h}^{-1}$ em Córrego do Ouro, GO (Oliveira et al., 2005) e 139,5 $\mathrm{mm} \mathrm{h}^{-1}$ em Alvorada, TO (Silva et al., 2003).

Pela distribuição espacial das estações utilizadas neste trabalho pode-se notar elevada concentração de estações na região do nordeste paraense, o que indica a necessidade de aumento do número de estações nas outras regiões.

As diferenças observadas entre as intensidades de precipitação contribuem para reforçar a necessidade de obtenção de equações de chuvas intensas para cada localidade de interesse. Uma das formas de minimizar as imprecisões na estimativa da intensidade de precipitação é aumentar cada vez mais o número de localidades estudadas.

\section{ConclusõES}

1. As equações de chuvas intensas foram ajustadas para 74 estações pluviométricas do Estado do Pará, com valores de coeficiente de determinação acima de 0,99. Ocorreu uma variabilidade dos valores de intensidade de precipitação pluvial, para uma mesma duração, entre os diferentes municípios estudados.

2. A maioria das estações $(51,4 \%)$ apresentou intensidade de precipitação entre 90 e $110 \mathrm{~mm} \mathrm{~h}^{-1}$, para uma duração de chuva de 30 min e um período de retorno de 15 anos.

\section{LITERATURA CITADA}

ANA - Agência Nacional das Águas. Hidroweb: Sistemas de informações hidrológicas. http://hidroweb.ana.gov.br. 15 Jul. 2011.

Back, A. J. Relações entre precipitações intensas de diferentes durações ocorridas no município de Urussanga, SC. Revista Brasileira de Engenharia Agrícola e Ambiental, v.13, p.170175, 2009.

Beijo, L. A.; Muniz, J. A.; Volpe, C. A.; Pereira, G. T. Estudo da precipitação máxima em Jaboticabal, SP, pela distribuição de Gumbel utilizando dois métodos de estimação dos parâmetros. Revista Brasileira de Agrometeorologia, v.11, p.141-147, 2003.

DAEE/CETESB. Departamento de Água e Energia ElétricaCompanhia de Tecnologia de Saneamento Ambiental. Drenagem urbana: Manual de projeto. São Paulo: DAEE/ CETESB, 1980.466p.

Damé, R. C. F.; Teixeira, C. F. A.; Terra, V. S. S. Comparação de diferentes metodologias para estimativa de curvas intensidade-duração-frequência para Pelotas-RS. Engenharia Agrícola, v.28, p.245-255, 2008.

Genovez, A. M.; Zuffo, A. C. Chuvas intensas no Estado de São Paulo: Estudos existentes e análise comparativa. Revista Brasileira de Recursos Hídricos, v.5, p45-58, 2000.

Longo, A. J.; Sampaio, S. C.; Suszek, M. Equação de chuvas intensas e precipitação provável para o município de Cascavel, PR. Revista Varia Scientia, v.6, p.119-127, 2006.

Mello, C. R.; Silva, A. M.; Lima, J. M.; Ferreira, D. F.; Oliveira, M. S. Modelos matemáticos para predição da chuva de projeto para regiões do Estado de Minas Gerais. Revista Brasileira de Engenharia Agrícola e Ambiental, v.7, p.121-128, 2003.

Mello, C. R.; Viola, M. R.; Mello, J. M.; Silva, A. M. Continuidade espacial de chuvas intensas no Estado de Minas Gerais. Ciência e Agrotecnologia, v.32, p.532-539, 2008.

Oliveira, L. F. C.; Antonini, A. A.; Griebeler, N. P. Estimativa de chuvas intensas para o Estado de Goiás. Engenharia Agrícola, v.28, p.22-33, 2008a.

Oliveira, L. F. C.; Antonini, J. C. A.; Fioreze, A. P.; Silva, M. A. S. Métodos de estimativa de precipitação máxima para o Estado de Goiás. Revista Brasileira de Engenharia Agrícola e Ambiental, v.12, p.620-625, 2008b.

Oliveira, L. F. C.; Cortês, F. C.; Wehr, T. R.; Borges, L. B.; Sarmento, P. H. P.; Griebeler, N. P. Intensidade-duraçãofrequência de chuvas intensas para algumas localidades no Estado de Goiás e Distrito Federal. Pesquisa Agropecuária Tropical, v.35, p.13-18, 2005.

Pfafstetter, O. Chuvas intensas no Brasil. Rio de Janeiro: Ministério da Viação e Obras Públicas; DNOS, 1957. 420p.

Pinto, F. A. Chuvas intensas no estado de Minas Gerais: análises e modelos. Viçosa: UFV, 1995. 87p. Tese Doutorado 
Pinto, F. A.; Ferreira, P. A.; Pruski, F. F.; Alves, A. R.; Cecon, P. R. Equações de chuvas intensas para algumas localidades do Estado de Minas Gerais. Engenharia Agrícola, v.16, p.91104, 1999.

Robaina, A. D. Modelo para geração de chuvas intensas no Rio Grande do Sul. Revista Brasileira de Meteorologia, v.4, p.95-98, 1996.

Santos, G. G.; Figueiredo, C. C.; Oliveira, L. F. C.; Griebeler, N. P. Intensidade-duração-freqüência de chuvas para o Estado de Mato Grosso do Sul. Revista Brasileira de Engenharia Agrícola e Ambiental, v.13, p.899-905, 2009.

Silva, B. C.; Clarke, R. T. Análise estatística de chuvas intensas na Bacia do Rio São Francisco. Revista Brasileira de Meteorologia, v.19, p.265-272, 2004.
Silva, D. D.; Gomes Filho, R. R.; Pruski, F. F.; Pereira, S. B.; Novaes, L. F. Chuvas intensas para o Estado da Bahia. Revista Brasileira de Engenharia Agrícola e Ambiental, v.6, p.362-367, 2002.

Silva, D. D.; Pereira, S. B.; Pruski, F. F.; Gomes Filho, R. R.; Lana, A. M. Q.; Baena, L. G. N. Equações de IntensidadeDuração-Frequência da precipitação pluvial para o Estado de Tocantins. Engenharia na Agricultura, v.11, p.7-14, 2003.

Silva, D. D.; Pinto, F. R. L. P.; Pruski, F. F.; Pinto, F. A. Estimativa e espacialização dos parâmetros da equação de intensidadeduração-frequiência da precipitação para os Estados do Rio de Janeiro e Espírito Santo. Revista de Engenharia Agrícola, v.18, p.11-21, 1999. 